\title{
Violencia familiar contra los niños: respuestas institucionales ${ }^{*}$ Domestic violence against children: institutional responses
}

\author{
Marie-Astrid Dupret**
}

\begin{abstract}
Resumen
A pesar de ser alarmante la tasa de incidencia de violencia contra los menores, este tema, por lo menos en el Ecuador, no ha sido objeto de una investigación científica seria, tanto en el plano cuantitativo como cualitativo, resultando raras veces pertinente la intervención; y tampoco la denuncia indiscriminada a las autoridades aporta una respuesta adecuada. Es urgente crear espacios de atención integral, que se encarguen de un trabajo con la familia, incluido el agresor o agresora; y en los casos más graves recurrir a una justicia restaurativa que tome en cuenta a las víctimas, así como a los victimarios. Será posible entonces evitar el ciclo de las revictimizaciones y proponer los lineamientos para una verdadera prevención.
\end{abstract}

\section{Palabras-claves}

Abuso sexual, maltrato grave, prevención, revictimización, violencia.

\begin{abstract}
Despite the alarming incidence of violence against children, at least in Ecuador, the issue had not been the object of serious investigation, neither in quantitative nor qualitative terms. Intervention only very rarely produces adequate results, and neither do indiscriminate complaints to law-enforcement authorities. There is an urgent need to set up instances for comprehensive support, in order to work with the affected families, including the aggressor, and, in the more serious cases, seek restorative justice that considers both the victims and the perpetrators. Only then it will be possible to avoid the circle of re-victimization and offer guidelines for genuine prevention.
\end{abstract}

\section{Key words}

Sexual abuse,severe ill-treatment / prevention / re-victimization / violence.

Forma sugerida de citar: $\quad$ DUPRET, Marie-Astrid. 2012. "Violencia familiar contra los niños: respuestas institucionales”, en: Universitas, enero-julio de 2012. Quito: Editorial Abya-Yala, pp. 17-51.

\footnotetext{
* $\quad$ Este artículo nace en el marco de una investigación intitulada "Intervención psico-social y jurídica para el tratamiento de la violencia intrafamiliar contra niños, niñas y adolescentes", financiada por la UPS.

** Doctora en Filosofía, psicoanalista e investigadora asociada del Centro de Investigaciones sobre Niñez, Adolescencia y Juventud, CINAJ, de la Universidad Politécnica Salesiana, UPS.
} 
La violencia contra niños y adolescentes, lejos de ser un fenómeno aislado y propio a una situación cultural particular, está íntimamente ligada a la desestructuración sociocultural de la posmodernidad y depende en gran medida de factores relacionados con la globalización, en especial por la destrucción de los valores tradicionales y éticos. Además, las agresiones ejercidas contra las nuevas generaciones dentro de la familia, constituyen la 'matriz' de la gran mayoría de las otras formas de violencia, de modo que entender su dinámica mortífera abre el camino a la aprehensión de las causas de las fuerzas invisibles que corroen la convivencia social contemporánea.

En sí misma, la violencia contra los más jóvenes es una realidad aterradora, y las investigaciones al respecto concuerdan para mostrar que este fenómeno no solo es muy común, sino que parece aumentar en un mundo donde las actuaciones agresivas, tanto en el plano físico como en el sexual se hacen cada vez más frecuentes (Informe 2009).

De ahí la urgencia de pensar en los medios necesarios para fomentar una verdadera 'prevención'. Y quien habla de prevención sabe que una política cuyo fin es atajar el fenómeno de la violencia contra niños, niñas y adolescentes, además de fundarse en principios éticos claramente definidos, tiene como requisito inicial estudiar bien la problemática en juego, comenzando por el contexto sociocultural en el cual esta violencia se produce, así como sus características, singulares y estructurales, en cuanto respuestas al fenómeno de desestructuración del lazo social propio a nuestra época; y como segundo requisito, cernir con mucho detenimiento la realidad del abuso sexual contra los menores, así como las formas graves de maltrato, un tema a menudo olvidado, a pesar de sus consecuencias mortíferas para la construcción psíquica del joven. De ahí una tipificación diferencial de los abusos sexuales y maltratos, además de una apreciación de su nivel de gravedad en función de sus efectos inmediatos, a mediano y a largo plazo, que servirán para dimensionar la problemática con mucho más precisión.

En un segundo momento se especificarán los pasos para una intervención pertinente y respetuosa, develamiento, señalamiento y denuncia, para luego proponer un breve recorrido por las instituciones a cargo de la educación y del cuidado de los niños y adolescentes: escuelas y colegios, centros de salud y de apoyo psico-social entre otros; por ser los principales lugares donde el maltrato y/o el abuso sexual intrafamiliar se visibilizan, lo que permite resaltar su responsabilidad muy especial en cuanto al develamiento de las situaciones de 
violencia intrafamiliar, a partir de lo cual se vuelve posible la implementación de las medidas necesarias para su tratamiento.

Unas palabras con respecto a las consecuencias de un imperativo de denuncia indiscriminada y de sus efectos negativos en la dinámica de un proceso terapéutico permitirá entender la importancia de fortalecer -e incluso crearespacios intermedios, con estructuras aptas para procesar de manera integral las situaciones de violencia y con una capacidad de involucrar en el proceso no solo al menor agredido sino a toda su familia, incluyendo al agresor o agresora: única manera para evitar la repetición de los maltratos y abusos.

Para completar este recorrido de los modos de intervención se expondrá sobre la Justicia, garante de la convivencia pacífica entre los miembros de una comunidad, y, por ende, garante en el seno de una familia. En nuestros tiempos posmodernos, a menudo la justicia aparece como un sistema rígido, cerrado, que se limita a la aplicación mecánica de leyes inertes, a pesar de haber consistido, desde los albores de los tiempos, en una discusión, un debate alrededor del acto culposo, abordado con sus circunstancias y en función de la persona acusada de cometer la acción ilícita o criminal. De ahí la aparición de una 'nueva' justicia, en realidad un retorno a una justicia 'humana', llamada justicia restaurativa que no sólo da un lugar primordial a las víctimas sino que también toma en cuenta al victimario, una justicia que, al considerar cualquier trasgresión o cualquier hecho que violente las leyes como una ruptura de la ética de convivencia pacífica de la comunidad, promueve soluciones nuevas como la mediación, cuyos fines últimos son la restauración de los vínculos socioculturales del grupo afectado.

Al mejorar las condiciones de intervención en las situaciones de violencia contra las generaciones más jóvenes y crear nuevas respuestas a los casos más acuciantes será posible atajar el círculo vicioso de las repeticiones que engendra inevitablemente una revictimización sin fin de los más vulnerables y vulnerados. Solo entonces, cuando se dé un paso al conocimiento de las causas psicológicas y sociales de la violencia ejercida contra niños y adolescentes dentro de la familia, y que se consoliden procedimientos eficientes y eficaces para invertir su curso, será posible pensar en las exigencias y los requisitos de una verdadera prevención, a la cual anhelamos todos. 


\section{Las causas socioculturales de la violencia familiar contra los más jóvenes}

Los comportamientos de abuso sexual y maltratos siempre reflejan el lugar y la dinámica mortífera de la violencia dentro de la sociedad donde se manifiestan; más aun son indicadores de un fenómeno de desestructuración sociocultural, como si los agresores estuvieran actuando bajo el efecto de una voluntad oculta que les domina, y cuyo fin sería destruir a las nuevas generaciones portadoras de la esperanza en el futuro, interrumpiendo de este modo el ciclo de la vida.

El término 'violencia' deriva del latín violare: tratar con violencia, en el cual se encuentra la raíz latina vis (vires) con el sentido de 'fuerza en acción', 'fuerza ejercida contra alguien'. La palabra 'violencia' destaca la utilización de una fuerza física. Por esto, un comportamiento violento es contrario a una relación de diálogo y lenguaje. Como decía Lacan, citado por Jean-Pierre Lebrun, "la violencia aparece donde se deshace la palabra" (Lebrun 2004, 273).

Lévi-Strauss (1958) observaba que lo que le da consistencia a la estructura de una sociedad humana reposa en diferentes formas de intercambio, entre las cuales el intercambio de palabras ocupa un lugar esencial. Y cuando cesa la posibilidad de vincularse a través del lenguaje, dentro de un código culturalmente definido, surge la violencia, el enfrentamiento, la dislocación de los vínculos entre las personas que interactúan. A la inversa, los discursos, los significantes, crean un lazo social y unen a los individuos aislados dentro de unidades más extensas cuya función es asegurar su supervivencia y su protección.

Esta oposición entre palabra y violencia nos parece esencial para la comprensión de los efectos del maltrato y del abuso sexual contra los más jóvenes, en cuanto estas actuaciones son inconciliables con la vida sociocultural de una comunidad humana. Son manifestaciones de ruptura del pacto social cuya función fundamental es mantener la convivencia pacífica. En este sentido, se puede decir que las agresiones contra los más vulnerables del grupo indican un malestar profundo, una desestructuración de los valores culturales, la expresión descontrolada de una pulsión mortífera.

Si examinamos cualquier forma de abuso sexual contra un niño, un ser que no está todavía maduro sexualmente, no es difícil justificar la interpretación de este acto como una trasgresión del lazo social. Como Durkheim (1897), Freud (1912-1913) y el mismo Lévi-Strauss (1947) lo han demostrado, la Ley de pro- 
hibición del incesto, al establecer una demarcación entre generaciones y definir relaciones permitidas y prohibidas entre los miembros de un grupo, constituye la ley primordial de la vida social humana. Es en este marco de ruptura de la ética que hay que entender el abuso sexual contra los más jóvenes como una violación -término emparentado al de violencia- de la ley fundadora de la sociedad. En este sentido, la perpetración de cualquier agresión sexual contra un menor encubre ineludiblemente un daño que afecta el orden social. De modo que las consecuencias de este actuar van mucho más allá del perjuicio físico, y sus efectos ponen en peligro la integración del sujeto en formación a una comunidad humana, y a su capacidad para asumir un lugar propio en la trama de intercambios de su grupo.

En cuanto al maltrato grave que se da en la familia, que atañe al desarrollo físico y psicológico del niño, también hay que entenderlo como una conducta destructora que dificulta grandemente la posibilidad de convivencia social del menor. Porque con este modo de tratar a los niños la familia se aleja de su función esencial que es asegurar el crecimiento sano y feliz de las nuevas generaciones, en manos de las cuales se encuentra el futuro de la vida humana, y cuyas experiencias relacionales y afectivas serán determinantes para la calidad del lazo social por venir. Más aún, el maltrato grave alimenta la potencial pulsión de la muerte, en contra de la pulsión de la vida y del eros cuya función es precisamente unir los elementos aislados de lo real y asegurar la conformación de una trama social capaz de sostener a cada individuo en su intento de supervivencia (Freud 1927). En este sentido, el maltrato grave, de la misma manera que el abuso sexual, trastoca el curso de la socialización, destruye el deseo de vida y dificulta la inserción de los jóvenes en una cultura creativa.

Este rodeo inicial por una interpretación de las conductas destructoras contra los niños, niñas y adolescentes en el seno de su familia, así como por las rupturas de una convivencia armónica, favorable al desarrollo individual y grupal, era imprescindible para mostrar que las problemáticas de abuso sexual y de maltrato no se limitan a casos particulares sino que apuntan a un asunto de sociedad y, por ende, requieren ser tratadas con el establecimiento de principios y lineamientos políticos adecuados para alcanzar una verdadera prevención que ponga fin a la tendencia destructora subyacente a la sociedad actual (Haesevoets 2008).

Conviene ahora detenernos un momento en los conceptos de maltrato (grave) y abuso sexual para intentar especificarlos, con el fin de evitar su utilización 
indebida, por un lado y, por otro, para definir con más adecuación su nivel de gravedad en cuanto a sus consecuencias no solo en el plan físico sino también psíquico.

\section{2. 'Maltrato grave' y 'abuso sexual' contra menores: ¿De qué hablamos?}

Para entender nuestro argumento es imprescindible precisar lo que cae dentro de lo que se llama violencia contra menores. Se puede proponer una definición psicoanalítica: es violencia el actuar de un adulto ${ }^{1}$ contra un niño, una niña o un adolescente, cuando este actuar está desprovisto de palabras humanizadoras (Dolto 1984), pronunciadas o no (pueden ser intenciones o comportamientos cargados de significación); es decir, un actuar carente de sentido que se manifiesta con gestos, acciones, frases, conductas, cuyo fin no es transmitir algún valor ético, algún aprendizaje educativo, algún afecto, sino un simple desfogue contra el más pequeño, tratándolo como mera cosa, sin que exista por parte del adulto un afán de hacerle un bien y de cuidar por su bienestar. Este perifraseo es necesario en la actualidad en un esfuerzo de evitar la dilución de la noción de violencia a falta de una delimitación clara, hasta el punto que el término puede perder todo sentido cuando todo se transforma en supuesto maltrato. Porque hay comportamientos equivocados, comportamientos de adultos frente a sus hijos que parecen cargados de agresividad y que, sin embargo, no tienen una connotación de violencia; al contrario, hay otros cuya meta expresada es hacer daño al pequeño; y hay otros que son destructores a pesar de no tener una apariencia violenta.

Nos limitaremos aquí en hablar de las dos mayores formas de agresión contra menores: el maltrato grave y el abuso sexual, por contener en sí todas las otras expresiones de conductas destructivas que toman como blanco a las generaciones en formación. Cabe notar que el maltrato grave y el abuso sexual corresponden a fenómenos muy distintos, aunque a menudo no existe una solución de continuidad entre los dos, el abuso sexual es a veces la transformación

$1 \quad$ No se tratará en estas líneas de la problemática de los adolescentes (e incluso niños) agresores, por ser un tema que merece un abordaje específico. 
de una conducta maltratante en una relación de promiscuidad sexual con rasgos amorosos, por lo menos en apariencia.

\section{Abuso sexual}

En primer lugar hablemos del abuso sexual, por ser considerado como la forma de violencia más típica contra los menores, aunque no sea siempre la peor expresión de agresividad, por más equivocado que haya que considerar este comportamiento. No vamos a entrar en las definiciones del abuso sexual, numerosas, pero por lo general insuficientes para analizar la problemática en juego cuando no se las relaciona con otros aspectos, tanto psicológicos como socioculturales.

La forma emblemática del abuso sexual es el incesto, y -ya lo hemos dicho- parece muy conveniente relacionar todos los comportamientos de naturaleza sexual entre adultos y niños como expresiones incestuosas, por cierto una doble generalización, en cuanto al sexo y en cuanto al parentesco. Porque el incesto constituye, con el homicidio ${ }^{2}$ la mayor trasgresión de las normas socioculturales. No existe sociedad en la cual no se encuentre restos de esta prohibición fundamental, base estructural de la convivencia y de las leyes humanas. Recordemos que la Ley Universal de Prohibición del Incesto conlleva una interdicción, tanto intergeneracional como de orden sexual; es decir, enuncia que la generación de los padres no debería mezclarse sexualmente con la de los hijos, y que, por otro lado, se supone que el acercamiento sexual entre hombres y mujeres de una misma familia no está autorizada, aparte de la pareja de los padres.

Uno entiende entonces por qué la promiscuidad sexual de un adulto con una niña ${ }^{3}$-más aún si pertenece a su círculo familiar- puede tener consecuencias negativas para el desarrollo psíquico de la joven, por dos motivos principalmente: el primero, porque este hombre que cumple una función de padre para la niña la trata como a un objeto sexual, a pesar de que ella no haya alcanzado la madurez ni fisiológica ni emocional en cuanto a su sexualidad, de modo que

2 Como veremos, el maltrato grave de cierta manera siempre tiene que ver con un deseo de muerte y, por ende, con una intención homicida.

3 Las consecuencias psíquicas de un incesto con una niña impúber resultan ser mucho más graves que con una adolescente (siempre y cuando no haya embarazo). 
ella es llevada a graves confusiones psíquicas, afectivas y conductuales (Ferenczi 1981). El segundo motivo con efectos negativos tiene que ver con la sociocultura, punto que cabe resaltar en una época en la cual las limitaciones al nivel sexual parecen anticuadas e intrascendentes. En efecto, la niña que establece con su padre una relación demasiado cercana al nivel sexual -y aunque sea simplemente al nivel sentimental-, no se encuentra en condición de establecer al mismo tiempo amistades normales con amigos de su grupo etario, precisamente los que podrían llevarla hacia una sexualidad normal, conforme a su edad, con intercambios afectivos entre pares.

La promiscuidad sexual padre-hija, desde el atentado al pudor hasta la consunción del acto sexual, es paradigmática de cualquier forma de abuso sexual, y puede servir de referencia al estudio del nivel de gravedad de este comportamiento reprensible. Es preciso distinguir tres aspectos cuando se quiere analizar sus efectos trastornadores:

- El acto cometido en el plano de lo real, es decir, la naturaleza (material) del acto transgresivo y sus circunstancias concretas, edad del menor, duración de la conducta inadecuada o delictiva.

- El valor simbólico del acto cometido, lo que corresponde a su apreciación desde un punto de vista sociocultural y colectivo, o sea la interpretación de este acto en el seno de una comunidad dada; lo que incluye desde luego la ética familiar promovida por el grupo al cual pertenece la menor.

- Las características imaginarias del acto cometido, sus efectos en la subjetividad de la menor y lo que se puede presumir de su vinculación al desarrollo psico-sexual ulterior de la niña o adolescente.

Al tomar en cuenta estas distintas facetas del abuso sexual, sus características y sus consecuencias a corto, mediano y largo plazo, tanto al nivel individual como en el plano colectivo, se evita una categorización unilateral y permite matizar las consecuencias, y por ende, los modos de intervención. 


\section{Maltrato grave}

El maltrato constituye la otra vertiente de situaciones de violencia intrafamiliar y, desde nuestro criterio, muy a menudo la más inquietante, la que siempre acompaña las peores formas de abuso sexual. En efecto, entre los fenómenos de violencia contra menores, el maltrato grave ocupa un lugar demasiado olvidado, casi oculto, como si su realidad sobrepasara lo aceptable y lo imaginable. De hecho, cuando uno se adentra en este campo, necesita mucha fuerza para no caer en el espanto: niños con marcas de quemadura, con lesiones inexplicables por un accidente natural, con fracturas repetidas, otros que están encerrados durante días enteros, que comparten su comida con animales. Estos cuadros, difíciles de mirar porque hacen presentes de alguna forma un lado inhumano reprimido en cada uno, producen a menudo una reacción, conocida entre los profesionales, que es la de cerrar los ojos frente a estas situaciones inaguantables (Crivillé 1986).

El maltrato grave, aunque solo en casos extremos llega a causar la muerte del menor, no deja de ser emparentado con el homicidio porque su meta, a menudo inconsciente, es la destrucción moral, física y/o emocional del joven (Haesevoets 2008). Sin embargo, cabe precisar que no todo maltrato conlleva este anhelo mortífero y, a pesar de su apariencia de violencia, hay casos, no raros, en los cuales se puede reconocer fines educativos, desde luego inadecuados, pero que no encubren un deseo de muerte hacia el menor.

Por esto, insistimos en la necesidad de hablar de 'maltrato grave' para referirnos a un maltrato que tiene que ser analizado como una expresión de violencia y de deseo de aniquilamiento contra un niño, una niña o un adolescente; y de este modo no extender esta calificación a cualquier actitud o actuación respecto de los más jóvenes, aunque tenga todas las apariencias de una agresión. En efecto, una educación responsable puede aparecer a veces como un limitante para los comportamientos del menor, al ir en contra de sus tendencias e impulsos naturales; sin embargo, hay que tener en cuenta que para que el pequeño ser humano en formación pueda estructurarse psíquica y socialmente es ineludible que se encuentre con reglas y normas, las que por lo general se concretan a través de sanciones (por supuesto, bajo ninguna circunstancia, estos castigos pueden poner en peligro el desarrollo físico y psíquico del menor).

En este sentido, no conviene confundir cualquier castigo, cualquier sanción, con una expresión violenta, y esto por una doble razón: por un lado, una 
extensión del término violencia (maltrato y abuso sexual) a toda conducta de adultos que sea sentida por los niños y adolescentes como un displacer, y como un freno a su libertad y un límite a sus actividades anheladas, conduciría a una condena de toda forma de educación y de toda exigencia de superación en pos de su formación como ciudadano responsable. Por el otro, tendría como efecto diluir la capacidad de intervención frente a actos realmente dañinos en contra de los niños, y cuyas consecuencias son destructoras para su proceso de estructuración físico y psíquico. Esta última situación se ha vuelto muy frecuente en este momento, porque se alienta la denuncia de cualquier intervención de los padres que limite la omnipotencia del joven. La consecuencia de este hecho en el campo que nos interesa, es decir el tratamiento de la violencia, es la disolución de la idea de maltrato, hasta el punto que, al no diferenciar entre los maltratos graves que causan daños duraderos al niño, por un lado, y conductas llamadas de manera equivocada 'maltratantes' en la medida que imponen restricciones necesarias a los comportamientos juveniles, el verdadero maltrato, este que perjudica gravemente al menor en su desarrollo emocional e intelectual, queda desapercibido. ${ }^{4}$

Por este motivo, es muy importante apreciar en su justa medida la gravedad de una situación de maltrato y analizarla de la misma manera que el abuso sexual, tomando en consideración sus distintas vertientes (Dupret 1997):

- La naturaleza de la acción cuestionada: su forma, su frecuencia, sus circunstancias (materiales), la fuerza utilizada, o sea, su realidad concreta, incluyendo la edad de la víctima.

- El impacto y las repercusiones emocionales y relacionales de la acción cuestionada; lo que abarca tanto el aspecto subjetivo como la vivencia del acto por parte del menor.

- La interpretación de la acción cuestionada y de la intencionalidad del adulto: ¿conducta educativa?, ¿castigo culturalmente avalizado?; o, al contrario, ¿un comportamiento aberrante, sin ningún fin formativo?, ¿un acto de fuerza incontrolado e inexplicable?, ¿una voluntad de domar al otro?

$4 \quad$ Muy raras veces en Ecuador el maltrato grave está tomado en consideración y tratado como una problemática específica que necesita una intervención muy bien pensada y apoyada por medidas de protección. 
Esto nos lleva a recalcar en el hecho que solo después de un análisis adecuado, amplio y documentado, es posible determinar si el acto violento es el resultado de un comportamiento sin intencionalidad malévola y que solo tiene la apariencia de maltrato; o una conducta inapropiada e involuntaria, equivocada, pero sin deseo de destrucción; o si debe ser calificado de maltrato grave $\mathrm{y}$, por ende, ser objeto de las intervenciones pertinentes en lo que se refiere al maltratante y que requiere una atención profesional para el menor, e incluso, a veces, su internamiento en un lugar protegido, con el fin de salvaguardarle de las agresiones de un medio familiar mortífero.

Ahora bien, se ha puesto el acento en los aspectos más sociológicos de la violencia intrafamiliar, pero eso de ninguna forma resta peso a sus consecuencias psicológicas. En breve, el abuso sexual tiene efectos innegables de deterioro sobre la construcción identitaria del joven, proporcionalmente al grado de trasgresión, mientras que el maltrato grave distorsiona su evolución emocional y su capacidad de empatía con los otros, dificultando el establecimiento de relaciones afectivas con su entorno.

\section{3. ¿Cómo intervenir en situaciones de violencia en la familia? Develamiento, señalamiento, denuncia}

Como acabamos de ver, la violencia en la familia contra las generaciones más jóvenes es una realidad ligada a la pérdida de los valores éticos del mundo de hoy, un fenómeno que se extiende a la par con la desestructuración sociocultural y la desintegración familiar contemporáneas. Por cierto, las formas y manifestaciones de este drama están cada vez más conocidas, y se conoce una serie de tratamientos para atajar y revertir los efectos destructores de estas situaciones dolorosas. Sin embargo, grandes dificultades se oponen al establecimiento de un modelo adecuado de intervenciones, imposibilitando en gran parte la creación de una verdadera política de prevención.

En el abordaje de la problemática del maltrato grave y del abuso sexual conviene distinguir tres momentos, según las necesidades de la intervención: el develamiento, a partir de la visibilización del actuar violento, el señalamiento $y$, por último, la denuncia que lleva a la judicializacion.

Aunque el maltrato y el abuso sexual infantil tienen lugar sobre todo en el ámbito familiar -o en lo que viene a remplazarlo en situaciones de desestructuración familiar-, lamentablemente, la familia, en el mundo posmoderno, 
escapa en gran medida de toda intervención de instancias oficiales, a diferencia de otras épocas cuando existía un control social no solo considerado como normal y normativo, sino aceptado por todas las partes involucradas. De modo que, actualmente, es principalmente fuera del ámbito familiar que se visibilizan casos de violencia intrafamiliar contra los más jóvenes, en primer lugar en los espacios educativos, así como en las instituciones de salud y de atención psicológica y social.

La visibilización de una situación de maltrato y/o de abuso sexual contra un menor es, como la palabra lo indica, el momento cuando se hace visible, o sea, aparece y se manifiesta fuera del lugar y del momento en que se produce. Muy especialmente, se observa en ciertos comportamientos típicos de los niños violentados en expresiones materiales como en sus dibujos o gestos, en su decir, en actitudes depresivas; es decir, en una serie de síntomas muy típicos y fácilmente descifrables para quien conoce la problemática. La visibilización debería inmediatamente alertar a los adultos en contacto con el menor para que se fijen con atención en él y puedan ayudar a un develamiento del caso de abuso sexual o maltrato grave, para permitir al niño comentar lo que pasa con este adulto, o bien, que el adulto, al darse cuenta del malestar del joven, inicie un procedimiento de investigación del caso para permitir una intervención, la más adecuada posible.

El develamiento, (Crivillé 1994, 38), remite al "momento cuando el conocimiento del abuso sexual sale del círculo familiar restringido y está reportado a un tercero". Este concepto, si se lo extiende a la cuestión del maltrato, nos parece de gran utilidad para definir una transformación esencial en lo que se refiere a la situación de violencia intrafamiliar, cuando un adulto exterior al círculo de la violencia esté informado de la agresión y que, de este modo, esta situación salga del círculo cerrado de la familia.

De hecho, muy raras veces alguien de la familia devela el maltrato o el abuso que está padeciendo un miembro del círculo familiar, al menos que obtenga un beneficio de este hecho, como ocurre en caso de divorcio y de discusión respecto a la tenencia de los hijos. Varias explicaciones pueden darse a esta situación, entre la cuales la primera es cuando el maltrato y el abuso sexual aparecen como hechos normales en un medio excluido de una verdadera inserción sociocultural -legalmente se habla de 'rusticidad' para referirse a gente que no ha sido socializada dentro de las normas socioculturales propias a toda comunidad humana-; esto se observa sobre todo en situaciones de gran marginalidad. 
Existen también ciertos grupos para los cuales el maltrato y el abuso sexual, no por desconocimiento de las leyes sino por laxismo moral muy típico del mundo posmoderno, se han transformado en un modo de vivir. En estos contextos, los sujetos no toman conciencia de la distorsión de sus lazos familiares, salvo cuando surge un acontecimiento inhabitual que hace tambalear el edificio, así construido sobre fundamentos frágiles.

El señalamiento. Después de la visibilización y del develamiento del maltrato grave y del abuso sexual contra menores, sería oportuno crear un momento intermedio antes de pasar a una denuncia que significa judicializar el caso. Proponemos llamar este segundo nivel el del 'señalamiento' porque se trataría de 'señalar' las situaciones de violencia intrafamiliar a instituciones especialmente dedicadas a la intervención psico-social para este tipo de problemáticas. El señalamiento sería entonces el momento en el cual la situación de maltrato grave y/o de abuso sexual contra un niño o adolescente está llevado al conocimiento de una autoridad competente que se hace cargo de su tratamiento adecuado. En efecto, en su gran mayoría, las instituciones que asumen responsabilidades en la educación y en la protección de los menores no tienen ni el personal suficiente, ni la capacitación, ni los recursos para brindar una atención integral a los niños que sufren agresiones graves en su familia, más allá de su rol fundamental en el proceso de develamiento de estos casos. Estos 'espacios de señalamiento' estarían reconocidos por el sistema judicial, en cuanto aptos para ofrecer una evaluación precisa y asumir la atención apropiada, siempre y cuando una intervención emergente no sea requerida.

La denuncia a las instancias judiciales competentes sería el último paso cuando se trata de intervenir en situaciones de suma gravedad, es decir, cuando la vida y/o el equilibrio psíquico del menor puedan estar corriendo un peligro inmediato. De hecho, existe para estos casos la posibilidad de internar al menor en un hospital pediátrico, y es necesario emprender sin demora las acciones legales y penales pertinentes.

Más adelante analizaremos más en detalle la utilidad de los espacios de 'señalamiento', así como el proceso de denuncia, en relación con las nuevas tendencias de la justicia actual. Sin embargo, conviene hablar del develamiento, y lo que significa para el sujeto menor; además hay que precisar los lugares donde se puede dar este momento clave en cuanto a la posibilidad de intervención. 


\section{Momento y lugares del develamiento}

Para que sea posible intervenir no basta que se visibilice una situación de violencia intrafamiliar; es imprescindible que se produzca su develamiento, o sea que los adultos asuman la realidad del hecho que está pasando y lo lleven al conocimiento de profesionales competentes. El develamiento, al permitir la apertura del cascarón familiar, reviste para el niño violentado un valor máximo y requiere la mayor atención.

Cabe distinguir dos posibilidades de develamiento: la primera es cuando el menor habla con algún adulto de confianza respecto a lo que está ocurriendo en su casa; la segunda surge de una observación atenta por parte de un adulto cuando está en contacto con el niño.

El menor devela espontáneamente la agresión de la cual es víctima. La niña, el joven, ha establecido una relación de confianza con su maestro, por ejemplo, y le cuenta algo que le preocupa, que le hace sentir mal y que de alguna forma le duele mucho. Si el maestro está atento, el niño va a sentirse suficientemente protegido como para contar lo que le está pasando. Es como si estuviera pidiendo una boya de salvación a un adulto con quien ha establecido una relación más sólida. Uno entiende entonces la importancia incalculable de que este adulto pueda ofrecer al menor un oído atento a lo que le dice, sin espantarse al descubrir una realidad que prefiere ignorar.

El adulto se percata de que el menor no está bien psicológicamente. A menudo el maestro que conoce a sus alumnos o el psicólogo en un Centro de Salud, o todo adulto que está sensibilizado a las maneras de comportarse de un niño, se percata fácilmente de las manifestaciones de un malestar inacostumbrado. En otros casos, la observación seguida de un menor permite darse cuenta que no está bien psicológicamente y que necesita urgentemente conversar con alguien que está en condición de escucharle. Hay que insistir sobre esta capacidad de observación que permite al adulto fomentar un develamiento y tenderle una mano al niño agredido para ofrecerle la posibilidad de poner en palabras la violencia que está sufriendo. Sin embargo, muchos factores intervinientes favorecen o, a la inversa, frenan la apertura de un adulto para oír el develamiento de estos hechos siempre muy dolorosos. De ahí, la necesidad de una capacitación adecuada de los profesionales, tanto en el plan psicológico como en el manejo de las herramientas técnicas y de los protocolos de remisión. 
Otro menor advierte a un adulto que un compañero está sufriendo una agresión en su familia. Por fin, cabe añadir una tercera posibilidad que surge cuando el develamiento tiene lugar gracias a la intervención de un tercero, un compañero, una amiga, que se encarga de poner al tanto al adulto responsable de la situación. Otra vez, esta situación requiere la sensibilidad del adulto que da crédito a las palabras de un menor y acepta hacerse cargo del caso en un primer tiempo e iniciar un proceso de develamiento si le parece indicado -es decir si tiene la sospecha de que se trata de una situación de agresión intrafamiliar-.

Lugares de visibilización y develamiento. Después de este comentario sobre el momento del develamiento, conviene dirigir ahora nuestra atención hacia los lugares donde es posible poner en evidencia las situaciones de violencia contra las generaciones más jóvenes. Por lo general, la familia misma está implicada en estos casos y, por otro lado, el niño violentado no es quien puede dar a conocer su sufrimiento, a menos que sea amparado por unos adultos (o por lo menos por unos jóvenes más maduros que él). A cambio, las instituciones que tienen bajo su responsabilidad la educación y la protección de los menores juegan un papel clave en la detección de las situaciones de agresión contra los menores. En efecto, la visibilización de casos de violencia intrafamiliar se produce comúnmente en los lugares frecuentados por los niños y adolescentes, en primer lugar, escuelas, colegios e incluso jardines de infantes; además, pueden presentarse cuando el menor acude a un centro de salud u hospital, o cuando recibe una atención psico-social. De ahí la responsabilidad muy grande de estas instituciones respecto al develamiento de las situaciones de violencia y, por ende, la posibilidad de sacar al joven de su secreto familiar de abuso y maltrato.

Las instituciones de salud. Existe en Ecuador una verdadera preocupación por parte de las autoridades sanitarias, así como por los mismos médicos y el personal de salud en la detección y prevención de situaciones de agresión contra los más jóvenes, por cuanto muchas veces es en la consulta médica donde el maltrato grave y el abuso sexual se visibilizan, ya que existen protocolos y folletos explicativos al respecto. Aunque uno de los limitantes de las propuestas oficiales y de las acciones recomendadas para tratar la violencia contra niños, niñas y adolescentes viene de la asimilación de las problemáticas de los menores a las de las mujeres, sin tomar en cuenta que las mujeres son adultas, tanto física como mentalmente, mientras que los más jóvenes son seres en formación que requieren imperativamente una escucha adulta para poder dar a oír su sufrimiento. 
Por otro lado, no raras veces, las instituciones de apoyo psicológico y social reciben a casos cuyo motivo de consulta está directamente ligado a violencias intrafamiliares. También puede ocurrir -y es un punto extremadamente complejo- que sea en el curso de la atención terapéutica que se visibilicen y develen situaciones de abuso sexual y/o maltrato grave. Volveremos sobre este tema al analizar el alcance de la confidencialidad.

De todos modos, es dentro de las instituciones educativas, donde se visibilizan la gran mayoría de los casos de maltrato y del abuso sexual contra menores; $\mathrm{y}$, por ende, los profesionales de la educación son a menudo los primeros receptores de las confesiones de los niños, y también, muy frecuentemente, los que pueden notar su malestar en el aula o en el recreo, y de este modo tener la facultad de abrir el camino a un develamiento. Más concretamente, los maestros y profesores juegan un papel de primer plano, porque mantienen con sus alumnos una relación que les permite notar, mejor que cualquier otra persona, los comportamientos inusuales, llamativos o inadecuados con los cuales las víctimas de violencia intrafamiliar ponen de manifiesto su malestar y su sufrimiento.

Sin embargo, y de manera paradójica, se ha puesto de relieve un cierto desinterés de muchos educadores para atender a los problemas de violencia intrafamiliar, lo que se explica en parte por la falta de una formación más profunda en materia de psicología, en parte por la voluntad de no crearse problemas. Recordemos aquí las palabras oídas por una practicante de psicología en una escuela de sector marginal: "Sabemos que estos niños viven en su familia situaciones con muchas agresiones de toda índole, pero no podemos hacer nada al respecto; y por lo tanto, nos tenemos que limitar al apoyo pedagógico", lo que muestra a la vez un serio desconocimiento de las consecuencias directas del abuso sexual y del maltrato grave en cuanto a la capacidad de un niño para estudiar y, por el otro, la opción del mínimo esfuerzo por parte del profesional de la educación, es decir, no tomar en consideración las facetas de la vida de sus estudiantes que no se ven directamente a la luz del sistema de enseñanza.

Además cabe notar que, en muchos planteles educativos, existe una tendencia relativamente extendida que les lleva a preferir no tratar los problemas de violencia intrafamiliar por miedo a problemas judiciales, o simplemente por facilidad (no tener problema con la familia o no entablar investigaciones que requieren algún tiempo y esfuerzo), o incluso por falta de sensibilización. Hay que añadir que, por lo menos en Ecuador, al nivel ministerial, todavía no se considera la violencia intrafamiliar como un asunto que releva de la Educación, 
sino más bien de la salud en general o del Ministerio de Inclusión Económica y Social.

\section{El imperativo de denuncia, en contraposición a la atención terapéutica}

Una de las mayores dificultades enfrentadas por los profesionales que trabajan en relación con la niñez y adolescencia, planteles educativos, hospitales y centros de atención psico-social es saber cómo proceder cuando descubren un caso de abuso sexual o maltrato grave. Por supuesto, hay una respuesta inmediata, indicada por el Código de la Niñez y Adolescencia, que es la denuncia judicial, sin más ni menos; una respuesta monolítica y a menudo inadecuada, si se analiza los resultados concretos de la atención a la problemática de la violencia contra menores (Carrión y Figueroa 2008). Las razones de tal ineficacia o inadaptación son múltiples y de varias índoles. Existen dificultades organizacionales, materiales y administrativas; pero también hay otras, inherentes a la misma problemática que se quiere resolver. Incluso, en los peores casos, la denuncia abrupta puede transformarse en el causante de una grave revictimización del niño. Como sea, el imperativo de denuncia, sin estudios complementarios, no contribuye a la creación de políticas de prevención a pesar de ser estas, el objetivo último del abordaje del maltrato y del abuso sexual contra menores, y reduce la modalidad de intervención a un procedimiento único que no toma en cuenta ni al sujeto agredido, ni a su contexto de vida, ni a las circunstancias del acto culposo, ni siquiera al bienestar de la sociedad. En fin, se puede afirmar que el recurso a la denuncia sin matices crea más problemas de los que soluciona.

Dos cuestiones se plantean aquí. La una torna alrededor del principio de confidencialidad y del secreto profesional, la otra tiene que ver con la posibilidad de elaboración de la vivencia de agresión intrafamiliar en la psicoterapia, antes de llegar a una denuncia. Empero, recordemos que, en situaciones donde la vida del menor está amenazada, es imprescindible actuar de forma inmediata, proceder a la denuncia con las autoridades competentes y tomar las medidas indispensables para asegurar la protección del niño, de la niña o del adolescente.

Confidencialidad y secreto profesional. La palabra 'confidencialidad' aparece solo dos veces en el Código de la Niñez y Adolescencia, mientras que ni siquiera se menciona el secreto profesional. En el artículo 80 se escribe: "Los exámenes médico legales a un niño, niña o adolescente se practicarán en estric- 
tas condiciones de confidencialidad y respeto a la intimidad e integridad física y emocional del paciente"; pero no se refiere a la confidencialidad en relación con el tratamiento terapéutico. En el artículo 317, que concierne a los adolescentes infractores, casi al final se indica que: "Los funcionarios judiciales, administrativos y de la policía, guardarán el sigilo y la confidencialidad sobre los antecedentes penales y policiales de los adolescentes infractores,...”. Es decir, que la confidencialidad, en su relación con el secreto profesional en los casos de violencia contra menores, ha sido ignorada por los redactores de este código.

La confidencialidad se relaciona con el pudor: el niño aprende que lo que dice no es para todos, que la verdad de lo que cuenta tiene mucha importancia y que, por ende, es necesario para él saber en quien puede confiar. Los psicólogos por lo general saben lo necesario que es insistir en la confidencialidad, es decir, explicar con claridad al niño que lo que dice es solo para él y para su terapeuta.

Para establecer lo que se llama 'transferencia', un elemento esencial en la eficacia de una interpretación psicológica, es imprescindible que el niño esté seguro que no solo sus palabras van a ser escuchadas sino que lo que dice quede en el espacio terapéutico, como algo que le pertenece porque expresa algo de su ser. En particular, los menores suelen desconfiar de los adultos que les dicen una cosa y luego hacen otra; como es a menudo el caso de sus padres que consideran que todo lo de su hijo, incluso adolescente, es de su propiedad; en estas circunstancias, resulta muy dificultoso para un niño estructurar una subjetividad suficientemente fuerte como para poder adquirir una posición propia y no volverse conformistas al responder a las expectativas de sus padres, sin correr riesgos. Recordemos por fin que la obligación del secreto profesional (Haesevoets 2003, 311ss) y de la confidencialidad, no debería limitarse a los terapeutas sino que debería extenderse a todos los que, en el contexto de su trabajo, se enteran de un hecho de maltrato grave o de abuso sexual; nunca puede ser un tema de chismes, sino que debe ser tratado inmediatamente con las autoridades competentes.

Proceso terapéutico versus denuncia. Cuando el terapeuta empieza a sospechar de una situación de abuso sexual o de maltrato grave, su manera de proceder y de continuar el tratamiento toma un nuevo rumbo. Desde luego, debe estar capacitado para intervenir en estas circunstancias, pero sería muy contraproducente tenerle atado por un principio de denuncia forzosa. En primer lugar, porque en la gran mayoría de los casos, perdería ipso facto la confianza de su paciente, de modo que la transferencia afectiva, el principal motor de la cura, se 
vería gravemente afectada. Por otro lado, levantar el sigilo del abuso sexual y/o del maltrato grave, ${ }^{5}$ por lo general, es una tarea lenta que solo puede realizarse de forma pausada y responsable hasta llegar en el momento oportuno a una denuncia judicial o, en casos menos graves, a un señalamiento a una institución capaz de llevar adelante una atención integral que involucre no solamente al niño sino a todo su grupo familiar, incluso al agresor o agresora, cuando se trata de un delito que no necesariamente pasa por la justicia penal.

Cabe añadir que, en ciertos casos, resulta muy difícil cerciorarse de antemano de la veracidad de los hechos narrados por el menor en el espacio terapéutico, y una denuncia errónea podría provocar un perjuicio sumamente grave al niño en terapia si se utiliza sus palabras para sostener alegaciones falsas (Van Gijseghem 1990). Por otro lado, si la denuncia está llevada a cabo de manera intempestiva, puede causar la desaparición del niño de los círculos terapéuticos, porque sus padres temen una persecución judicial y, como consecuencia muy indeseable, su exclusión del único espacio de escucha del cual ha podido beneficiarse hasta entonces; por ende, el resultado obvio de un fin abrupto de la atención terapéutica es un nuevo encierro en el drama familiar.

Esta reflexión debe llevarnos a proponer vías alternativas para abordar la violencia intrafamiliar contra menores, además de mostrar el interés de un análisis más detenido del conjunto de la problemática que significa un maltrato grave y/o abuso sexual cuando involucra a las generaciones más indefensas.

\section{La necesidad de espacios de señalamiento y de atención in- tegral a la familia}

La atención integral a la familia. El interés de tener instituciones especializadas en el tratamiento de la violencia intrafamiliar contra menores es múltiple. Citemos algunos ejemplos de entre los más relevantes:

1. Disminuir la acumulación de casos en la Fiscalía y otros organismos judiciales.

2. Vigilar por la continuidad del proceso de atención.

$5 \quad$ No raras veces, el menor no recuerda nada de la situación de agresión aunque confiesa tener un sentimiento de malestar al respecto. 
3. Reducir los casos que no se develan por miedo a una judicializacion excesiva.

4. Ofrecer una atención terapéutica que incluya a la familia.

5. Proponer un trabajo psicológico con el agresor.

6. Ofrecer terapias alternativas y, muy en especial, una verdadera mediación familiar.

7. Facilitar un acompañamiento durante el proceso de atención.

8. Asegurar un seguimiento de los casos después de que hayan sido solucionados.

9. Por fin, $-\mathrm{y}$ tal vez sea lo esencial- permitir un abordaje novedoso de las agresiones dentro del entorno familiar, abriendo el camino a un refuerzo de la estructura del hogar en cuanto grupo esencial para promover un desarrollo sano y feliz de los más jóvenes.

Expliquémonos punto por punto.

1. El número de casos acogidos por la Fiscalía y otros organismos de la Justicia está en aumento constante y las estructuras actuales no dan abasto (muchos casos no pueden ser atendidos por falta de espacio). Un sistema de filtro y de orientación sería de gran beneficio para desatascar a las instancias judiciales y devolverles una mayor eficiencia. La otra ventaja sería evitar una judicialización excesiva al permitir intervenciones y procedimientos alternativos para situaciones que no son de suma gravedad, aunque, al prolongarse, sin beneficiar, de alguna forma la intervención, pondrían en riesgo el bienestar psíquico y físico del menor.

2. La otra ventaja que podrían ofrecer los espacios de señalamiento sería asegurar la continuidad de los procesos emprendidos, en cuanto los profesionales tendrían la posibilidad material de involucrarse personalmente en las causas que asumen y de este modo evitar la deserción, tan frecuente en los casos de maltrato grave y abuso sexual contra menores. Se podría considerar responsabilidad de los centros de señalamiento vigilar por la continuidad de los procesos y, cuando hay defecciones, tener la ayuda de la DINAPEN para encontrar a los niños desaparecidos del circuito de atención. 
3. El temor a la Justicia y el miedo a consecuencias desfavorables para el entorno familiar, ${ }^{6}$ constituye una reacción muy común en el entorno del menor violentado que impide el develamiento. El ofrecimiento de una atención más integral, más personalizada, adaptada a las circunstancias particulares, así como soluciones variadas que tomen en cuenta cada problemática específica, reducirían grandemente el número de casos que quedan en el secretismo de la familia, los más perjudiciales para las jóvenes víctimas.

4. Otro gran beneficio de la creación y reforzamiento de centros reconocidos oficialmente y especializados en el tratamiento de los casos de violencia intrafamiliar es que estos espacios deberían estar organizados para atender al grupo familiar en su conjunto, y no solo a la víctima. La integración de los distintos miembros del hogar en el trabajo terapéutico no solo es recomendable sino que constituye una exigencia ineludible si se quiere fomentar soluciones y remedios a largo plazo. Todos los profesionales que tratan cuestiones de abuso sexual y maltrato grave saben perfectamente que son males y patologías que suelen transmitirse de una generación a otra; y que para atajar esta repetición, es imprescindible incluir también a los hermanos, además de los adultos, en el trabajo terapéutico. En este sentido, la psicología sistémica que, como lo indica su nombre, se interesa a la familia en cuanto sistema, ofrece aportes valiosísimos al respecto.

5. Otro tema, relacionado al anterior, es la atención al agresor. La violencia intrafamiliar dibuja un círculo vicioso de repeticiones que se transforma fácilmente en comportamientos reiterativos que se hacen casi normales dentro del ámbito familiar. Muy a menudo, los agresores han vivido historias de gran violencia en su infancia; y en su gran mayoría son tanto víctimas como victimarios, de modo que necesitan urgentemente una atención especializada, no solo en el plan humano -son personas que sufren- sino también para proteger a la sociedad contra nuevos actos 
destructores. No hay política de prevención sin un trabajo terapéutico con el agresor.

6. Una característica de los centros que podrían servir como espacios de señalamiento es que usualmente proponen distintas alternativas terapéuticas en función de cada caso particular. Esta gama de procedimientos de atención tiene como ventaja adaptarse a la necesidad de cada uno de los usuarios. Para unos, una terapia personal les va a ofrecer por primera vez en su vida la oportunidad de ser escuchados en su sufrimiento subjetivo; para otros, el trabajo frente a frente es demasiado angustiante, por lo menos en un principio, y necesitan primero un espacio de palabra grupal. La mediación familiar entendida, en los casos que nos interesan, como el proceso de elaboración de la problemática de la violencia dentro de la familia, y de restitución de la palabra y del diálogo en lugar del silencio, en búsqueda de un compromiso de las partes en vista de una convivencia más armoniosa, constituye un instrumento de valor incalculable, incluso para la víctima que puede beneficiarse del reconocimiento del daño sufrido por parte de su agresor.

7. Los espacios de atención integral, por lo general, no tratan a sus usuarios como números, sino que suelen acogerles e integrarles a sus distintas actividades, dándoles la posibilidad de encontrar una forma de inserción sociocultural, un modo de atención como los que se encuentran a menudo en los centros que dependen de instituciones religiosas. Cabe recordar aquí que tanto el abuso sexual intrafamiliar como el maltrato grave constituyen rupturas muy fuertes de la convivencia social, y a la vez indican, por lo general, alguna forma de exclusión social. Una de las funciones insospechadas de los espacios de señalamiento podría ser la restauración del lazo social, muy deshilachado en los casos de violencia intrafamiliar.

8. Cabe añadir que existe otra necesidad para las familias dónde se han dado situaciones de violencia contra los más jóvenes, y de trasgresión de las leyes cuya función era mantener una convivencia familiar pacífica, es la de un seguimiento a mediano y largo plazo. Empero, de lo que sabemos, no existe este tipo de vigilancia -de veeduría- que asegure que las medidas implementadas y las terapias personales y grupales no 
pierden su efecto sanador después de cierto tiempo. El primer argumento para justificar este seguimiento a mediano y largo plazo es que una familia que ha sufrido un daño en su seno, debido a la actuación agresiva de uno o varios de sus miembros, pone en evidencia una fragilidad que persistirá por años, lo que explica por qué quienes se hayan enterado de estas situaciones tendrán que mantenerse en alerta para prevenir el surgimiento de nuevos casos de violencia a futuro. El segundo argumento a favor de un seguimiento durante varios años es la tendencia tan específicamente humana a la repetición. Esta repetición en las pautas de los comportamientos -y que se da con especial insistencia en circunstancias dolorosas como si se tratara de un esfuerzo absurdo para entender lo que está pasando- no solo es propia de los agresores sino que atraviesa las generaciones, como un legado inarticulable en palabras, y que se transmite por una reiteración sin sentido.

Notamos respecto a este último punto dos cosas: Por un lado, el seguimiento de ninguna forma debería limitarse a los casos atendidos en los espacios de señalamiento sino que habría que extenderlo a todas las familias que han necesitado una intervención psico-social y/o judicial. En otros países existe una expresión específica para este apoyo de larga duración, se llama "Acción educativa en medio abierto". Por otro lado, cuando hay situaciones de gran precariedad, debería existir la posibilidad de incluir a estos hogares en programas de esta índole, con el fin de prevenir un deterioro a futuro de la vida familiar y de las relaciones entre padres e hijos.

9. Por último, si se considera a las familias como el eslabón esencial que media entre el niño y la sociedad, debido a sus responsabilidades para asegurar el bienestar físico y mental, así como el desarrollo sano de los más jóvenes, las instituciones que asumen la misión de trabajar con estos grupos de parentesco, con el fin de disminuir la violencia en su seno, cumplen una función clave en un mundo cada vez más desorientado en cuanto a los valores éticos y sociales y a las reglas de convivencia pacífica. Más que nunca, los adultos reclaman apoyos y consejos en sus tareas educativas; a la vez muchas distorsiones en las relaciones familia- 
res, muchos tratos inadecuados se explican por el desconocimiento de los principios básicos de las funciones parentales, y de su desautorización paulatina. Por este motivo, los lugares y centros que pueden brindar una asesoría en este campo, se han vuelto muy útiles y logran a menudo disminuir la espiral de la violencia, permitiendo a la familia reorganizarse como hogar acogedor, protector y formador del devenir sociocultural de los jóvenes. En este sentido, es mejor evitar una intervención judicial, cuando esta herramienta radical no se impone como la única posibilidad para restablecer el orden de la vida en sociedad -y en familia-.

Todos estos argumentos abogan a favor de la creación o del reforzamiento de espacios especializados que sirvan para el señalamiento de situaciones de agresiones intrafamiliares y que tengan las condiciones y la formación necesaria para brindar una atención integral, ofrecer una escucha personalizada y proponer soluciones concretas a las problemáticas encontradas.

En el Código de la Niñez y Adolescencia, Título V, se habla de "Los organismos de ejecución del sistema nacional de protección” y, del Art. 209 al 214, se define su naturaleza y se desglosan sus funciones. Son las 'entidades de atención' que "tienen a su cargo la ejecución de políticas, planes, programas, proyectos, acciones y medidas de protección y sanción, de acuerdo a las políticas y planes definidos por los organismos competentes y a las instrucciones de la autoridad que legitimó su funcionamiento." (Art. 209). Y se menciona como primera obligación de dichas entidades de atención: "Promover las relaciones personales directas con la familia e impulsar actividades que permitan el fortalecimiento del vínculo o la reinserción familiar en el menor tiempo posible, según los casos." (Art. 211 a). Y la segunda recomienda: "Realizar acciones educativas con los familiares al cuidado del niño, niña o adolescente". (Art. 211 b).

Aunque escuetos, con pocas precisiones técnicas y sin objetivos definidos, estos dos párrafos justifican, promueven y apoyan toda acción destinada a incorporar a la familia en los procesos que involucren a los menores para obtener una mejoría de sus condiciones de vida. Por lo tanto, la facultad de crear espacios de señalamiento y de fomentar programas de atención integral está incluida en el Código de la Niñez y Adolescencia, y bastaría mejorar la organización de las 'entidades de atención' en función de un conocimiento de la problemática familiar más elaborado e investigado. 
Porque no es suficiente decir que se va a trabajar con los hogares, es necesario conocer de más cerca las dificultades estructurales de las familias en general y, en particular, las de las de los sectores desfavorecidos, y sus carencias específicas en cuanto a educación y cultura, cuyas falencias han conducido a las situaciones de violencia contra los menores que se han visibilizado y que han sido develadas por las instituciones responsables de curar el malestar de los niños. Dicho de otro modo, si se quiere evitar una repetición de los dramas de los niños violentados, es imprescindible trabajar en la reeducación y el reforzamiento de la estructura familiar, elemento fundamental para asegurar el crecimiento sano tanto en el plano material como en el intelectual y el emocional de las nuevas generaciones. Dicho de otro modo, no hay defensa de los niños y adolescentes que no pase por un trabajo con la familia, incluyendo a los hermanos y al agresor o la agresora.

Para completar el estudio sobre las modalidades de intervención, hay que decir unas palabras sobre el sistema judicial, el último recurso en el abordaje de los casos de violencia, el más radical, que permite dictar sentencias e imponer sanciones.

\section{Justicia restaurativa: en contra de la tendencia hacia la hi- perjudicialización}

No hay sociedad que pueda prescindir de una Justicia, uno de los tres poderes cuya acción es esencial en el mundo actual, al lado de los poderes legislativo y ejecutivo. Sin embargo, la Justicia no constituye una institución fija y eterna; y cada época ha implementado una jurisprudencia en base a sus principios éti$\cos \mathrm{y}$ a sus necesidades funcionales.

Justicia restaurativa y (re)habilitación. A pesar de los imperativos de seguridad y de represión que están muy de moda, la época presente asiste al surgimiento de una nueva tendencia en la Justicia que privilegia un abordaje del crimen o delito desde una perspectiva personalizada, y que incluye en primer lugar a la víctima y a su demanda de reparación, pero también al agresor (u ofensor) a quien se da la posibilidad de reintegrarse a su comunidad, mediante una posibilidad de reparación. Esta nueva justicia se llama Justicia restaurativa (o reparadora). Sin entrar en mayores detalles, lo que quisiéramos recalcar aquí son dos aspectos que nos parece de gran relevancia, tanto en lo que se refiere a la intervención judicial en los casos de maltratos y de abusos sexuales contra 
menores, como en cuanto al lazo social que sostiene no solo a cada grupo sociocultural sino a cada sujeto en su singularidad.

El primer aspecto novedoso en la justicia restaurativa es el lugar acordado a la relación de palabra entre la víctima y su victimario, lugar de valor esencial para los niños para quienes lo que se dice, la enunciación verbal, tiene un altísimo peso estructurante. Que un adulto reconozca con palabras el daño cometido, tiene efectos infinitamente más fuertes que cualquier castigo que a la larga crea en la víctima infantil un enorme sentimiento de culpabilidad.

El segundo aspecto deriva de la oportunidad de reinserción social para el agresor. Porque se considera que, si él manifiesta un auténtico deseo de reparar los daños causados, se le debe abrir la posibilidad de reintegrarse a su grupo social, y por ende a su familia, siempre y cuando asuma su (re)habilitación y se comprometa en trabajar a favor de la restauración del lazo social y afectivo que él mismo ha roto con su trasgresión.

Último punto, no hay que pecar por ingenuidad y pensar que bastan unas palabras de arrepentimiento para que el agresor esté comprometido en un proceso de reparación. Es un trabajo de larga duración, lo que subraya la importancia de un seguimiento a mediano y largo plazo, una necesidad esencial en el buen funcionamiento de una Justicia restaurativa.

Aunque todavía no tiene mucho espacio en Ecuador, y menos aun en lo que se refiere a delitos y crímenes sexuales, la Justicia restaurativa, si se utiliza con todas las garantías necesarias, marca un gran progreso frente a una justicia que se limita a castigar y encerrar en prisiones, lugares que muy a menudo se vuelven las mejores escuelas del crimen. ${ }^{7}$ Además, los principios de la Justicia restaurativa tienen efectos más allá del ámbito del Derecho y trazan nuevos horizontes en cuanto a una transformación de los valores socioculturales hacia una dimensión más humana, en oposición a la hiperjudicialización de la posmodernidad que excluye a los actores, transformándose en una mecánica de represión y de sanción indiscriminada. Porque la justicia puede dar lugar a una nueva forma de violencia; dejemos la palabra a Elías Neuman, en su libro Mediación penal $(2005,124)$ :

7 Todos los estudios al respecto indican que los agresores sexuales, en particular contra los niños, viven una situación carcelaria de suma violencia y humillación; y los suicidios no son raros, con las consecuencias fácilmente imaginables para sus hijos. 
"Los juicios penales resultan agresivos, inmisericordes y destructivos, en el sentido de que quiebran todo vínculo e implican, en el plano social, una profundización de la crisis. [...] Distancian al objetivo esencial del derecho, que es la concordia, y no logran los fines propuestos que la ley pretende inspirar".

Para completar este corto comentario respecto a la Justicia restaurativa, parece oportuno hablar de la mediación, un instrumento de resolución de conflictos fundado en el vínculo de lenguaje entre víctima y victimario, bien diferente de la negociación.

La mediación, una resolución alternativa de conflictos. La 'mediación' como procedimiento alternativo de resolución de conflictos es un modelo de justicia de gran antigüedad que ha sido recientemente reintroducido dentro del nuevo modelo de Justicia restaurativa; y en la medida que se funda en un diálogo dirigido, en presencia de un tercero como garante, se adecua plenamente a la búsqueda en la cual se inscribe esta investigación, cuyo objetivo es mejorar los modos de intervención en situaciones de violencia intrafamiliar, con el fin de promover políticas de prevención del maltrato y del abuso sexual contra niños, niñas y adolescentes, y por ende, mejorar la convivencia sociocultural. Existen varios tipos de mediación: familiar, escolar, civil, y también penal, donde todas responden a un mismo principio que es restablecer los lazos entre los distintos actores del drama de la violencia gracias a la palabra. De este modo la mediación se presenta como una alternativa a la aplicación drástica de una justicia impersonal. Se la puede definir entonces como un intento de "escuchar a las partes y confrontar sus puntos de vista para permitirles encontrar una solución al conflicto que les opone (...), y restablecer el diálogo entre ellas, con el fin de lograr acuerdos constructivos" (Benedicto 1999, 22).

Todavía faltan mucho más trabajos respecto a la mediación en casos de violencia intrafamiliar; sin embargo, se puede adivinar su valor incomparable para tratar los conflictos que involucran a menores en relación con los adultos que han desistido de sus responsabilidades parentales, y que de cierta manera les han engañado con una palabra mentirosa y una actuación reprensible en el plan ético. No es el lugar para profundizar los aspectos técnicos de la mediación y sus exigencias morales, pero cabe insistir en la importancia de incluir la mediación familiar en los casos menos serios de agresiones contra los más jóvenes y, en los más graves, la mediación penal, bajo la supervisión de un juez, por constituir procedimientos muy valiosos en el abordaje y el tratamiento de la violencia intrafamiliar. 
Antes de cerrar estos párrafos sobre la Justicia restaurativa, conviene mencionar otro instrumento de la intervención judicial en las situaciones de abuso sexual contra niños -y que podría ser, mediante algunas adaptaciones de mucho provecho para los casos de maltrato grave-, que es la entrevista única, una técnica que, también, se fundamenta en el uso estructurante de la palabra y, más específicamente, en el respeto al decir de los niños.

La entrevista única. No es nuestra intención presentar la Entrevista Única de manera detallada sino sugerir una mayor utilización de este protocolo en Ecuador, siempre y cuando se lo implemente con todas las condiciones y garantías de una aplicación ética y profesional (INNFA 2002). Desde hace algunos años, existe en EE.UU. una red constituida por distintos actores comprometidos con la protección de los niños y adolescentes contra el abuso sexual y el maltrato. Con el fin de asegurar una atención óptima a los menores víctimas de abusos y evitar su revictimización por estar sometidos a exámenes y sobretodo indagaciones repetitivas y a menudo agresivas, han elaborado un acuerdo interinstitucional respecto a un 'protocolo de entrevista única' para determinar los procedimientos óptimos y definir la información idónea para obtener un documento con validez legal y que sirva de prueba forense (Coulborn Faller 1993).

El objetivo del protocolo de la Entrevista Única es reducir el sufrimiento y la manipulación del niño y, eventualmente, de su familia en el juicio de abuso sexual, disminuyendo el número de interrogatorios necesarios. Además, permite mejorar la investigación pericial de los hechos ocurridos con una información obtenida con más garantía de veracidad y con el mayor respeto a la palabra del joven, limitando también las posibilidades de afabulación y de manipulación del menor por adultos interesados en distorsionar su decir.

Cabe insistir en el hecho de que no todo adulto, ni siquiera todo profesional de la infancia está capacitado para conducir una Entrevista Única, en cuanto se apoya en una técnica y una ética específicas. Sin embargo, tanto en lo que se refiere a la Mediación Familiar como a la Entrevista Única, sería de mucho provecho habilitar a ciertas instituciones cuya seriedad en su dedicación a la atención psicológica, social y jurídica, a situaciones de violencia contra los menores, está comprobada, pero que no pertenecen directamente al sistema judicial, para que ofrezcan estos servicios a la comunidad, y así ayuden a aliviar el amontonamiento de trabajo en las fiscalías y juzgados de la niñez. Precisamente, los espacios de señalamiento serían los más aptos para implementar una 
utilización óptima de estos instrumentos destinados a fomentar la Justicia más humana, que es la Justicia restaurativa.

\section{El peligro de la revictimización}

A estas alturas de nuestra reflexión es imprescindible añadir unas palabras más sobre la cuestión de la revictimización de los menores en los procedimientos psico-sociales y jurídicos, un tema que solo se mencionó de paso hasta ahora, un tema de mucha actualidad en vista de las dificultades de articulación y remisión entre las distintas instituciones a cargo del bienestar y de la protección de los menores.

Como la palabra lo indica, se llama revictimización a una reiteración de la agresión contra la víctima. La revictimización se da en circunstancias de distinta índole, como la multiplicación de exámenes periciales, interrogatorios, tests y pruebas. Otra forma de revictimización es el fin de no recibir de muchas instituciones que delegan a otros la responsabilidad de asumir el caso de violencia. Lo que todos estos casos de revictimización tienen en común es el hecho de tratar al niño como un objeto desechable, no como un sujeto que está sufriendo, y la ignorancia de su sensibilidad, manteniéndole en un desconocimiento de lo que le está sucediendo, la peor forma de revictimización siendo la falta de respeto a su palabra y a su manera de expresar lo vivido, o también la utilización de un discurso crudo que no preserva su imaginario infantil. A la luz de las investigaciones, la revictimización aparece como moneda muy común en la atención a los casos de violencia contra los niños, niñas y adolescentes, por carencias en la formación de los profesionales en el campo de la infancia $-\mathrm{y}$ esto se observa a todo nivel de la atención, pero tal vez con más nitidez aun en la función judicial (y la policía), porque hay pocos abogados y jueces preparados para tratar con niños y niñas. También a menudo existe entre los adultos el temor de dejarse involucrar en los dramas familiares que esconden la mayoría de los casos de abuso sexual y maltrato grave (Crivillé 1986).

En este sentido, es imperativo siempre escoger, entre todas las opciones de intervención de las cuales disponemos, la menos agresiva contra el menor, la que toma más en cuenta sus necesidades esenciales y su bienestar (superior, como se enuncia en el Código de la Niñez y Adolescencia). Bajo ninguna circunstancia, se justifica una intervención que perjudique al niño, a la niña o al 
adolescente en su equilibrio psíquico, mental y relacional, por más que responda a las exigencias del sistema social o judicial.

\section{Hacia una política de prevención}

Hemos hablado de la problemática de la violencia ejercida contra niños, niñas y adolescentes por parte de familiares adultos, y de la manera más adecuada de intervenir frente a estos casos. Es posible ahora formular propuestas concretas para atajar y evitar la repetición de estas situaciones mortíferas, e incluso pensar en políticas que permitan revertir estos fenómenos de desagregación social y sus consecuencias desastrosas sobre el devenir de los más jóvenes; en suma, hablar de prevención, cuyo objetivo inmediato es disminuir drásticamente las peores formas de violencia contra las generaciones más indefensas.

El concepto de 'prevención' se entiende como 'acción y efecto de prevenir'; y también: 'preparación o precaución para evitar un riesgo'. Al nivel semántico, la palabra viene de 'prevenir', un verbo con varias acepciones, entre las cuales: preparar y disponer con anticipación las cosas para un fin; prever, conocer de antemano un daño o un perjuicio; precaver (con el sentido de evitar un daño o peligro), evitar o impedir una cosa; evitar o vencer un inconveniente, dificultad u objeción; y también, resolver una dificultad.

En Psicopatología del Niño (De Ajuriaguerra y Marcelli 1989) se toma en consideración tres niveles de prevención:

La prevención primaria, que "apunta al mejoramiento del medio de vida del niño, con el fin de impedir la aparición de trastornos. Interviene al nivel del entorno familiar o social".

La prevención secundaria, que "consiste en detectar lo más pronto posible los trastornos, con el fin de evitar que se estructuren bajo un modo patológico fijo".

La prevención terciaria, que "intenta evitar o atenuar las secuelas o las complicaciones de un estado patológico constatado y evitar el paso a la cronicidad". 
Respecto al tema de la violencia intrafamiliar, por lo general, prevalece el sentido de prevención primaria, un esfuerzo para prevenir o evitar la ocurrencia de algo, como las situaciones de maltrato grave y violencia. Aunque no se suele hacer referencia a aquellas, la prevenciones tanto secundaria, como terciaria, debería ser el objeto de mucha atención, con la implementación de medidas adecuadas para hacerlas efectivas en la realidad. La prevención secundaria tiene como objetivo detectar lo más pronto posible las situaciones familiares que puedan engendrar alguna expresión de violencia contra los más jóvenes en el proceso de develamiento: es la función de las instituciones que tienen a su cargo a menores y cuya importancia se ha dicho aquí. En cuanto a la prevención terciaria, su rol esencial es impedir una repetición de hechos dañinos. Esta repetición puede darse en dos momentos muy distintos. El uno ocurre en el presente inmediato; en este caso, el fin de la prevención, es evitar que el maltrador o maltratadora, o el abusador o abusadora, vuelva a cometer la misma acción dañina contra el niño, la niña o el adolescente. Pero también hay otro momento, más tardío, cuando el menor, ya más grande -aunque, a veces, todavía adolescente-- repite la situación de violencia que ha experimentado en su infancia; esta repetición transgeneracional de las situaciones de abuso y maltrato obliga a pensar en una prevención específica, ligada con el trabajo terapéutico y, sobre todo, con un seguimiento a mediano y largo plazo para estas familias que han evidenciado una gran fragilidad en su dinámica psico-social.

Una serie de preguntas se plantean, en función de cada uno de los tres niveles de prevención. Enumeremos algunas:

1. ¿Cuáles son las medidas y las acciones que conviene implementar para impedir situaciones de maltrato grave y abusos sexuales? Este aspecto apunta a la función de las instituciones a cargo de niños y adolescentes, dentro de un sistema de prevención.

2. ¿Por qué ciertas personas están llevadas a actuar en contra del bien de los niños y adolescentes, a pesar de tratarse de familiares cercanos? Remite a la faceta psicológica y subjetiva del maltrato y del abuso sexual contra menores.

3. ¿Cuáles serían las causas que originan situaciones de maltrato y abuso sexual contra los más jóvenes? Aquí se plantea la necesidad de analizar los procesos en juego, situándonos en un campo sociológico y antropológico. 
4. Por fin, a todo nivel, y atravesando cualquier pregunta respecto a la violencia intrafamiliar, está el tema de la familia: ¿Qué es una familia? ¿Cuál es su papel en la estructuración subjetiva de los menores? ¿La familia monoparental puede cumplir con su rol en la formación de los más jóvenes? ¿Y las nuevas familias? Estos son algunos de los temas que deberían estar sometidos a una investigación profundizada, desde distintas disciplinas.

Porque el establecimiento y la puesta en marcha de políticas de prevención implica, en primer lugar, la definición de los lineamientos éticos y socio-culturales de la sociedad en la cual se quiere intervenir, en particular a los principios que sustentan el concepto de familia por un lado, y por el otro, la idea de educación y de formación de los más jóvenes. Solo a partir de este delineamiento, será posible precisar objetivos claros para los programas y proyectos de atención a las situaciones de violencia intrafamiliar contra niños, niñas y adolescentes; lo que a su vez permite precisar y concretar las acciones pertinentes para cumplir con estos objetivos, o por lo menos acercarse a las metas propuestas. El último paso necesario para la implementación de una verdadera política de prevención es que no puede ahorrarse una evaluación muy exigente y detallada de los resultados, tanto en cuanto a los logros como a los fracasos y errores.

A partir de esta base se podrá pensar en el establecimiento de una verdadera política de prevención orientada por un conocimiento serio de las problemáticas socio-familiares y psicológicas en juego y de las consecuencias nefastas de intervenciones intempestivas y/o indebidamente sustentadas profesionalmente, tanto como, a la inversa, del silencio cómplice de adultos que optan por mantener el secretismo y el aislamiento emocional de los jóvenes más indefensos, encerrados en situación de violencia.

\section{Conclusiones}

Tanto el maltrato grave como el abuso sexual no son dos figuras cualquiera de violencia, sino transgresiones de las dos leyes fundadoras de la sociedad: la prohibición del incesto y la prohibición de matar, y por ende, comportamientos destructores del lazo social. Sin embargo, se observa un gran desconocimiento de la realidad del maltrato grave y del abuso sexual contra los menores, que se 
torna evidente cuando se analizan los datos referentes a estas problemáticas en Ecuador. No existen registros precisos de esta problemática; ningún estudio ofrece una estadística detallada de las situaciones de maltrato grave y las últimas investigaciones respecto al abuso sexual (Informe 2009) se basan en estudios a posteriori, a partir de adultos entrevistados sobre asuntos de abusos sexuales sufridos en su infancia.

La atomización de las acciones, proyectos y programas para intervenir en casos de violencia contra niños y adolescentes constituye uno de los mayores escollos para lograr una verdadera prevención. El Código de la Niñez y Adolescencia, casi la única referencia para el tratamiento de la violencia contra menores, adolece de un desarrollo suficiente en el abordaje de estas problemáticas, apenas mencionadas en el texto. El resultado es que cada institución intenta resolverlas de la mejor manera posible, pero sin un sustento teórico y/o práctico suficiente.

Al nivel superior de intervención, desde las instituciones del Estado, se observa una situación comparable a raíz de la desarticulación de las diversas modalidades de abordaje. Ninguna de estas instancias se considera responsable de la formulación de las políticas socioculturales para las nuevas generaciones; y menos aun, autorizada para reclamar por el incumplimiento de las metas de protección integral de los más jóvenes. La dificultad del diálogo con las instancias judiciales es un inconveniente grave para un mejor acompañamiento y seguimiento de los casos; aunque ha surgido en los últimos meses un esfuerzo para enfrentar esta situación (Consejo de la Niñez y Adolescencia 2011). Tampoco existe una concertación sostenida entre los ministerios encargados de asuntos relativos a la niñez y adolescencia, lo que impide una aproximación más eficaz a estas problemáticas.

La problemática de la violencia contra los más jóvenes, no se puede diluir en capítulos y subcapítulos de otras políticas, sino que la definición de los lineamientos de la política hacia la infancia y la adolescencia debería ir mucho más allá del Código de la Niñez y Adolescencia y debería proceder del trabajo de especialistas de la infancia, psicólogos y psicoanalistas, educadores, médicos, sociólogos, trabajadores sociales, y con el asesoramiento de expertos en cuestiones de justicia. 


\section{Bibliografía}

BENEDICTO, Anita

1999 “La médiation familiale judiciaire: Obligation ou libre élection entre les parties?”, en: Dialogue 143: Justice et Familles, Questions Ouvertes. Primer Trimestre.

CARRIÓN, M. y A. Figueroa

2008 Del incesto: discursos, realidad y procedimientos jurídicos. Tesis. Quito: Pontificia Universidad Católica de Ecuador.

Consejo de la Niñez y Adolescencia

2011 "Encuesta a Juzgados de la Niñez y Adolescencia 2011".

COULBORN, Kathleen

1993 Child Sexual Abuse: Intervention and Treatment Issues. United States, Department of Health and Human Services Administration for Children and Families.

CRIVILLÉ, Alberto

1986 "Los profesionales frente a los padres que maltratan a sus hijos", en: II Congreso Latinoamericano sobre Maltrato al Menor. Guayaquil, Ecuador 1621 de noviembre del 1985.

DE AJURIAGUERRA J. y D. Marcelli

1989 Psychopathologie de l'enfant. Paris: Masson.

DOLTO, Françoise

1984 L'image inconsciente du corps. Paris: Seuil.

DUPRET, Marie-Astrid

1997 Preliminares para una encuesta sobre el maltrato infantil. Propuesta de investigación sobre el maltrato infantil presentada a la Fundación Desarrollo y Autogestión DYA e Instituto del Niño y la Familia INFA, Junio 1997 (Revisado en 2010). No publicado.

DURKHEIM, Émile

1897 La prohibition de l'inceste et ses origines.

FERENCZI, Sandor

1933 "Confusión de lengua entre los adultos y el niño. El lenguaje de la ternura y de la pasión", en: Psicoanálisis, tomo IV.

FREUD, Sigmund

1912-1913 Totem y Tabu.

1927 El porvenir de una ilusión, tomo VIII: 2961-2992 
HAESEVOETS, Yves-Hiram

2003 Regard pluriel sur la maltraitance des enfants. Vade mecum didactique, éditions kluwer, Bruxelles.

2008 Traumatismes de l'enfance et de l'adolescence. Un autre regard sur la soufInforme 2009 france psychique, Éditions De Boeck Université, Bruxelles.

2009 Investigación sobre abuso sexual a niñas, niños y adolescentes en el Ecuador. Ministerio de Inclusión Económica y Social del Ecuador, Instituto de la Niñez y la Familia - INFA, Defensa de los Niños Internacional - DNI Ecuador.

INNFA, Sistematización d las Conferencias del Equipo de San Diego y los Protocolos de para la Atención en Maltrato y Abuso Sexual en Niños y Adolescentes (La Entrevista Única), Quito.

LEBRUN, Jean-Pierre

2004 Les désarrois nouveaux du sujet. Paris: Erès.

LÉVI-STRAUSS, Claude

1947 Las estructuras elementales del parentesco. Buenos Aires: Paidos.

1958 Anthropologie structurale. Paris: Plon.

NEUMAN, Elías

2005 Mediación penal. Buenos Aires: Editorial Universidad.

REGALADO, Diana

2010 ¿Es justificable desde un punto de vista psicológico, una mediación penal en caso de incesto? Disertación para la obtención del título de psicólogo clínico, Quito, PUCE. No publicada.

VAN GIJSEGHEM, Hubert

1990 "Facteurs interférant avec la qualité du témoignage de l'enfant au tribunal dans des causes d'abus sexual", en: Revue canadienne de psycho-éducation, Vol. 19, No. 1, pp. 11-22.

Envío 27 de febrero de 2012 - Fecha de aceptación 26 de marzo de 2012. 